\title{
Therapeutic hypothermia in middle-income settings: Where to now?
}

Worldwide, deaths related to complications around the time of birth (intrapartum-related deaths), are the third leading cause of under 5-mortality. ${ }^{1}$ Globally, each year, an estimated 1.15 million infants develop NE with around a quarter of survivors affected by long-term neurodisability. ${ }^{1}$ The majority of this burden falling on the poorest of countries.

In this issue, Kali et al. describe the early outcomes of infants with hypoxic-ischaemic encephalopathy (HIE) after introduction of therapeutic hypothermia (TH) to their neonatal service in the Western Cape province of South Africa. ${ }^{2}$ Therapeutic whole body cooling to a core temperature of $33.5^{\circ} \mathrm{C}$ for 72 hours, is standard of care in many high income countries (HICs) with evidence of increased disability-free survival and improved cognitive outcomes at early school age. However, evidence from low- and middle-income settings is limited, with the only systematic review of TH in this setting (567 infants, 7 studies) failing to show a significant reduction in neonatal mortality. ${ }^{3}$

One of the great strengths of the study by Kali et al. is that the outcomes that they report are from a 'real life' cohort from a middle-income setting. ${ }^{2}$ In doing so, they have effectively highlighted many of the challenges faced in translating evidence and protocols from HIC studies to a mid-resource setting, where important differences in population and care setting frequently exist. Ideally, an RCT examining the effectiveness of $\mathrm{TH}$ would be conducted, however, a normothermic study arm is surely hard to justify in a setting where neonatal intensive care is available, and where the authors themselves have previously shown TH to be safe and feasible. ${ }^{4}$

An important factor highlighted by this study is the differences in definition of HIE between different resource settings that may influence early outcomes. Definitions of HIE used in HIC cooling trials may be difficult to replicate where limitation or lack of diagnostic tools such as cord gas measurement and aEEG are less common. Whilst Kali et al. successfully met common cord/blood gas criteria, early aEEG, used to define 'moderate/severe' encephalopathy for inclusion in the Toby study, was clearly not always available, and difficulties around quality control are also highlighted. Perhaps as a result, around a third of infants receiving TH were ultimately defined as mildly encephalopathic and therefore would not meet current criteria for TH in HICs.

Differing aetiology of HIE may also effect outcomes in different settings. High delivery rates, lower staff to patient ratios and delays in transfer time more common in lower resource settings are likely to affect the nature and timing of insult and may mean that the narrow therapeutic window for early intervention has passed. Different prevalence's of important co-morbidities such as infection and hypoglycaemia may also affect outcome.

What is clear is that more research from middle-income country settings is desperately needed if we are to facilitate our understanding of how TH protocols may be safely and effectively introduced in middle-income settings, so that in the future many more newborns can safely and effectively benefit from this life changing intervention.

\section{References}

1. Lee AC, Kozuki N, Blencowe $\mathrm{H}$, et al. Intrapartum-related neonatal encephalopathy incidence and impairment at regional and global levels for 2010 with trends from 1990. Pediatr Res 2013; 74 Suppl 1: 50-72

2. Kali GT, Martinez-Biarge M, Van Zyl J, Smith J, Rutherford M. Therapeutic hypothermis for neonatal hypoxic-ischaemic encephalopathy had favourable outcomes at a referral hospital in a middle-income country. Acta Paediatrica, 2016; In press 
3. Pauliah SS, Shankaran S, Wade A, Cady EB, Thayyil S. Therapeutic hypothermia for neonatal encephalopathy in low- and middle-income countries: a systematic review and meteanalysis. PLoS One, 2013;8(3):e58834

4. Kali CT, Martinez-Biarge M, Van Zyl J, Smith J, Rtherford M. Management of therapeutic hypothermia for neonatal hypoxic ischaemic encephalopathy in a tertiary centre in South Africa. Arch Dis Child Fetal Neonatal Ed. 2015; 100:F519-F523. 